\section{CABLE-SUSPENDED HAPTIC INTERFACE}

\author{
Robert L. Williams II \\ Ohio University, USA
}

Editor: Mel Siegel

\begin{abstract}
A cable-suspended haptic interface (CSHI) concept is presented. The goal is to create an input/output device to provide six-degree-of-freedom (dof) wrench (force and moment) feedback to a human operator in virtual reality or remote applications. Compared to commercially-available haptic interfaces for virtual reality applications, the present concept is driving for lighter, safer, crisper, more dexterous, and more economical operation. The CSHI concept is presented, along with the required mathematical transformations for use of the device.
\end{abstract}

\section{Introduction}

Haptic interfaces provide a sense of wrench (force/moment) and/or tactile feedback to the human from a computer-simulated or remote environment. Most existing wrench-reflection devices are jointed robots whose actuators are coordinated to provide a force (and some also provide moment) vector to a human user's hand (for example, the six-degree-of-freedom (dof) Kraft mini-master of Fig. 1 and the six-dof $J P L$ force-reflecting hand controller of Fig. 2). These devices are too heavy, strong, expensive, and not dexterous enough to achieve widespread application. Some commercial devices have appeared more recently. The three-dof PHANToM (Fig. 3) [Massie and Salisbury, 1994] is a jointed device with reasonable weight but provides only three Cartesian forces (no moments) to the operator's finger tip. The two-dof Impulse Engine 2000 (Fig. 4, Immersion Corp.) is an active flight stick which provides only two Cartesian forces via the flight stick handle.

The force-reflecting device proposed is an extension of two recently-developed technologies in cable-suspended robots and stringed haptic interfaces. A pioneer in cablesuspended robots is NIST with the Robocrane of Fig. 5 [Albus, et. al., 1993]. This device is similar to a six-dof Stewart's platform [Stewart, 1966], with cables instead of hydraulic-cylinder legs. The Stewart's platform, consisting of a six-dof moving table controlled by six hydrauliccylinder legs, has been widely applied as motion platforms for flight simulators. In Fig. 5, the lower triangle is a rigid body and the remaining lines are cables which can independently provide six tensions on the rigid triangle. The upper dashed triangle indicates a rigid support structure. In this system, gravity is as an implicit actuator which ensures cable tension is maintained at all times. Robocrane has been proposed for use in shipping ports.

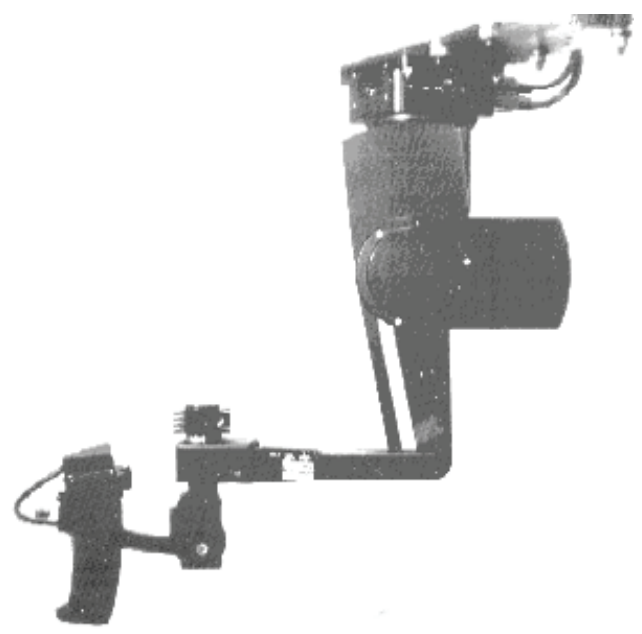

Figure 1: Kraft Mini-Master controller.

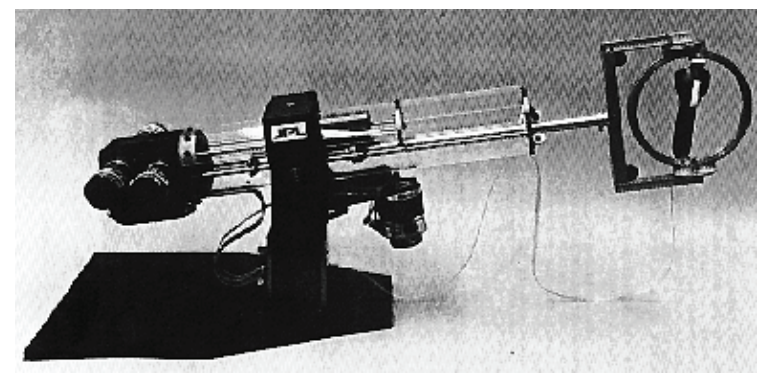

Figure 2: JPL Force-Reflecting Hand Controller

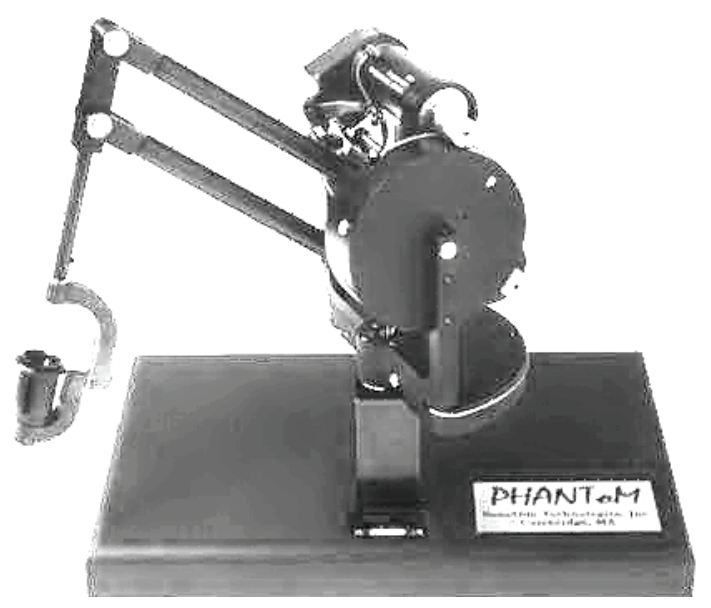

Figure 3: PHANToM

The cable-suspended robot Charlotte (Fig. 6) was developed by McDonnell-Douglas [Campbell, et. al., 1992]. Charlotte has flown in a Space Shuttle experiment and is currently used at NASA JSC for astronaut training. Fitted with an end-effector, it was designed for use as an 
automated microgravity experiment tender for International Space Station. The author assisted researchers at NASA Kennedy Space Center during the summer of 1996, adapting the Fig. 6 concept for post-flight inspections of the Space Shuttle radiator bays.

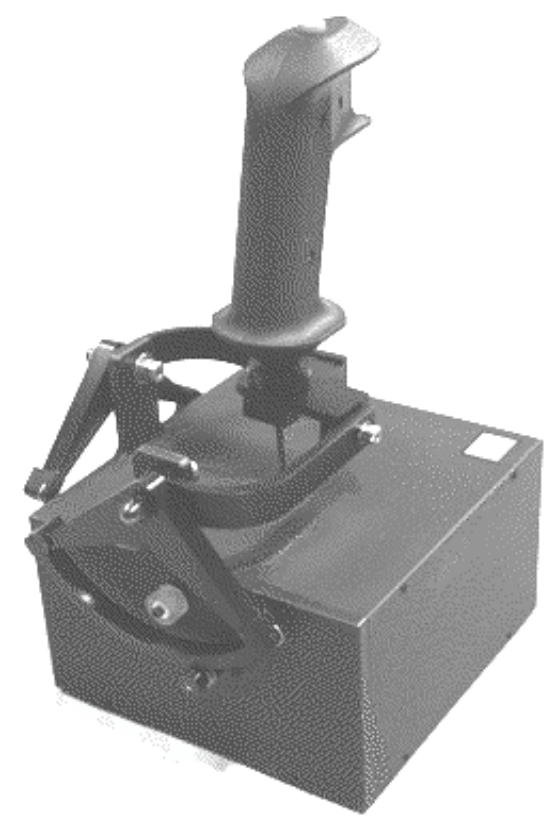

Figure 4: Impulse Engine 2000

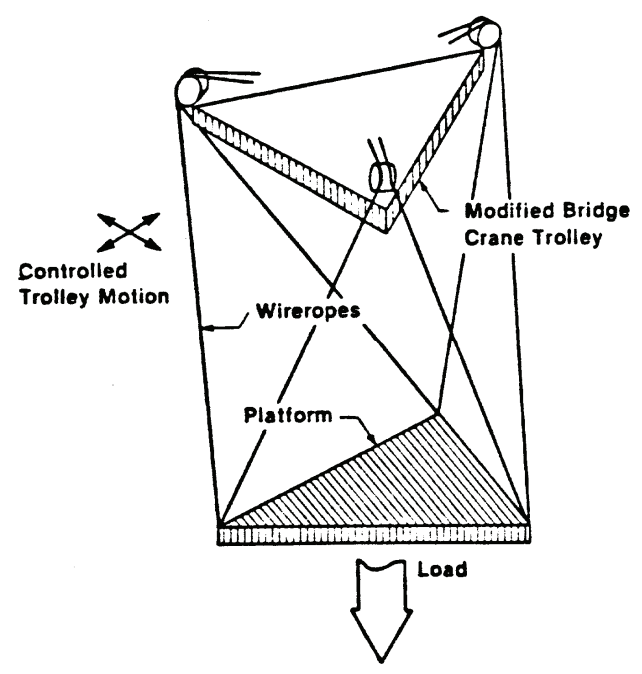

Figure 5: Robocrane

Two stringed haptic interfaces have been built and tested, the Texas 9-string [Lindemann and Tesar, 1989] and the SPIDAR [Ishii and Sato, 1994]. The Texas 9-string device was too bulky, suffered from cable interference, and failed to provide small feedback forces due to large actuator friction. Also, the bandwidth was low, which resulted in large time delays and jerky motion. The SPIDAR system was developed with four strings to give force-reflection to a single operator's finger tip (like the PHANToM). It was extended to eight strings to include thumb feedback.

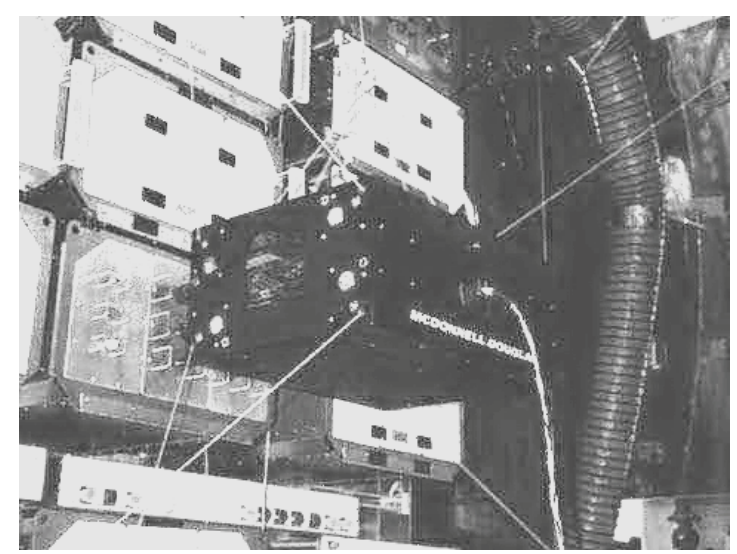

Figure 6: Charlotte Robot

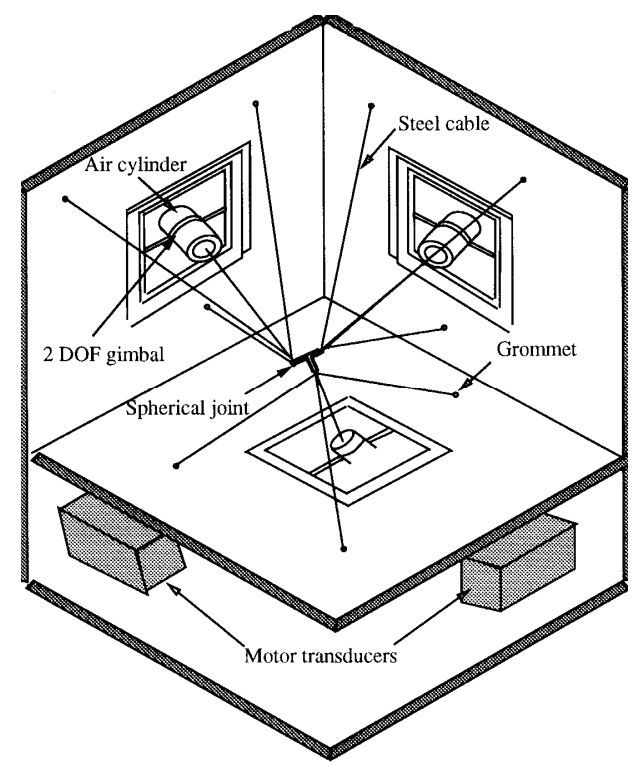

Figure 7: Texas 9-String

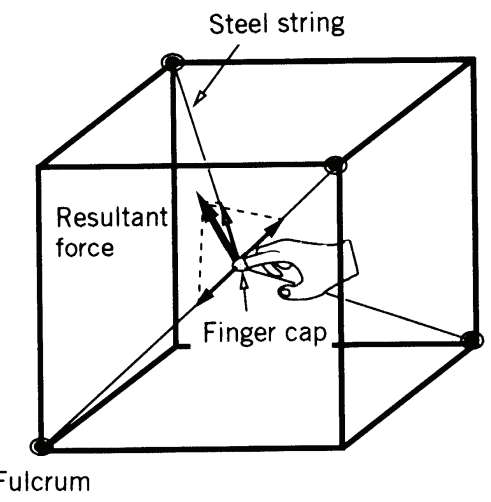

Figure 8: SPIDAR 
The current paper presents a concept for a six-dof cable-suspended haptic interface. Wrench-reflection is the current focus, although future plans include fusion of wrench/tactile feedback with the proposed interface. First the concept is presented, followed by required mathematical transformations for implementation of the device; lastly, the device control is presented and a discussion of VR applications is given.

\section{Haptic Interface Concept}

This section presents the cable-suspended haptic interface $(C S H I)$ concept. The CSHI consists of a hand-grip supported in-parallel by $n$-cables controlled by $n$ independent tensioning actuators, shown in Fig. 9. Each cable actuator system includes a torque motor, cable reel, tensioning mechanism, plus cable length and force sensors. For six-dof spatial operation, there must be at least six cables. Since cables can only exert tension on the hand-grip, there must be more than six cables to avoid configurations where the hand-grip can go slack. Figure 9 shows eight cables independently controlled by eight actuators mounted to the frame of the device. This scenario represents actuation redundancy but not kinematic redundancy. That is, there are two extra motors which provide infinite choices for applying six-dof wrench vectors, but the hand-grip has only six Cartesian-dof ( $x, y, z$, roll, pitch, yaw).

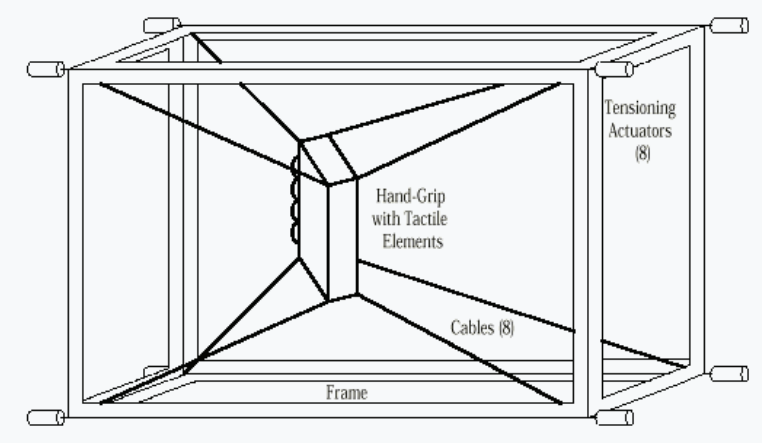

\section{Figure 9: Cable-Suspended Haptic Interface (CSHI) Concept}

A planar version of this concept has been developed with four cables and planar $(x, y$, twist $)$ motions. Figure 10 shows a photograph of the planar CSHI prototype developed by the author and research assistant Trevor Blackann. This device has served as proof-of-concept in hardware, but the quality is not yet sufficient to make meaningful comparisons with the existing haptic interfaces reviewed in the previous section. The motors are too large and easily overpower the human hand. It was learned that forces in any direction may be readily applied; however, for effective moments (twists) to the human hand, the cables should connect to opposite corners (as in the Charlotte hardware, Fig. 6). This lesson and many other from this planar prototype hardware will help determine the specifications and nature of the spatial prototype hardware, currently under development.

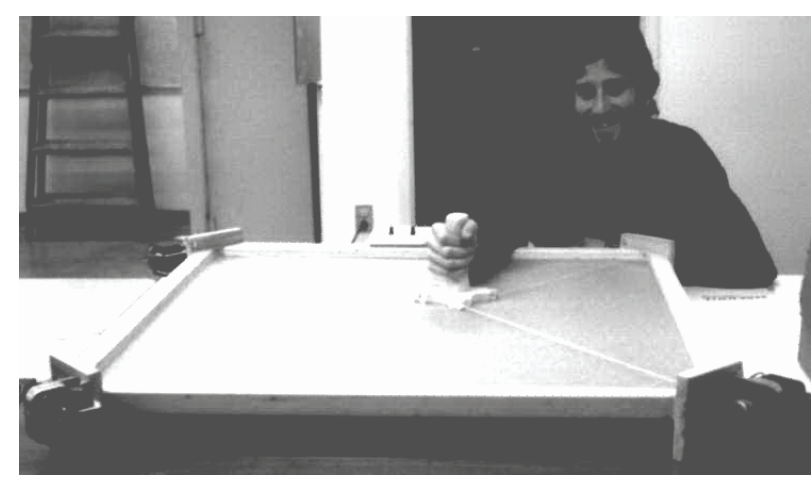

Figure 10: Planar CSHI Prototype

This haptic interface can be used for both input and output. The pose (position and orientation) of the hand-grip may be calculated and used to command the pose of objects in the virtual/remote world. Velocity commands to virtual objects are also possible, either by interpreting a static handgrip pose as a constant velocity or by tracking the velocity of the user's hand. The device is used as output when reflecting wrenches to the human user. Simulated object weight, mass moment of inertia, stiffness, dynamics loads, and environment contact forces may thus be felt. The next section gives the forward kinematics, inverse Jacobian matrix, and statics modeling required for operation of the CSHI.

\section{Mathematical Transformations}

Kinematic modeling is concerned with relating motions of the hand-grip with motions of the cables. The forward pose kinematics solution is required to calculate operator commands from the CSHI, while the inverse Jacobian matrix and statics modeling are required to command wrenches to the hand-grip. These models are non-linear and configuration-dependent.

Figure 9 presents the spatial and Fig. 11 the planar CHSI kinematic diagrams. Figure 9 shows crossed cables, while Figs. 10 and 11 show non-crossed cables. The methods in this section can be applied to either crossing case. The crossed-cable case may have moment-applying advantages over the non-crossed case. The moving frame is $\{H\}$ and the fixed frame is $\{0\}$. The cable index is $i$, $i=1,2, \cdots, n$. The dimension of the Cartesian space is $m$. The number of cables in these examples are $n=8$ and $n=4$ for the spatial and planar cases, respectively. Since cables can only exert tension, generally actuation redundancy $(n=8>m=6$ for spatial and $n=4>m=3$ for planar) is required to ensure no cables are slack during use. The ground link cable connection points are $G_{i}$ and the moving link cable connection points are $H_{i}$. Vectors $h_{i}$ give the locations of $H_{i}$ relative to the origin of $\{H\}$. The cable lengths are $L_{i}$. The hand-grip point of interest is the origin 
of $\{H\}$. The kinematic equations derived in this section apply to any general spatial or planar $C S H I$.

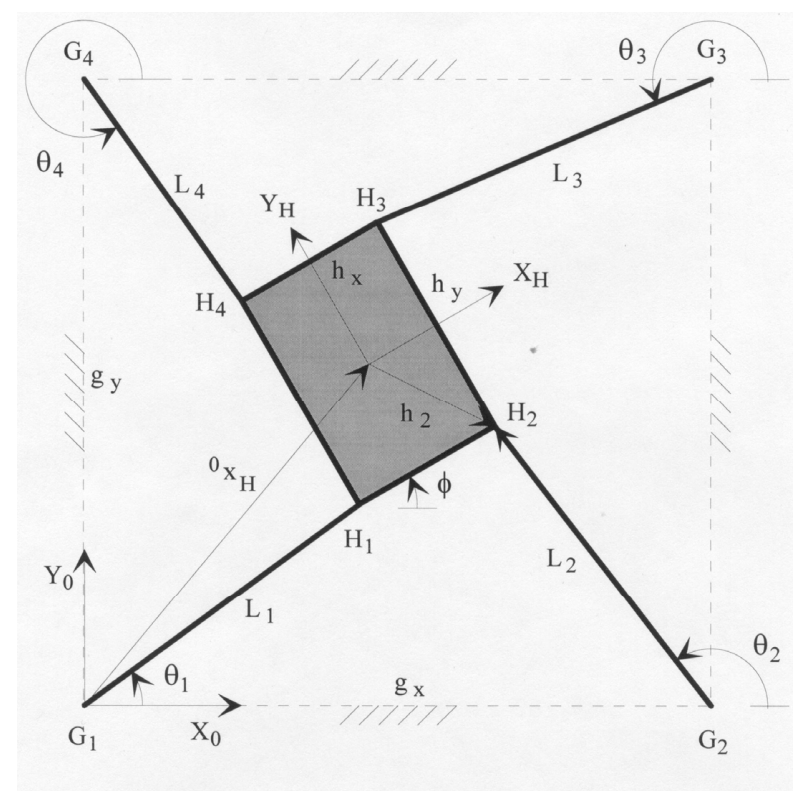

Figure 11. Planar CSHI Kinematic Diagram

\subsection{FORWARD POSE KINEMATICS}

The hand-grip pose is described by ${ }_{H}^{0} T$ (position ${ }^{0} x_{H}$ and orientation ${ }_{H}^{0} R$, using $Z-Y-X \alpha, \beta, \gamma$ Euler convention, [Craig, 1989]):

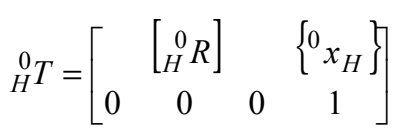

$$
\begin{aligned}
& { }_{H}^{0} T=\left[\begin{array}{cccc}
c \alpha c \beta & -s \alpha c \gamma+c \alpha s \beta s \gamma & s \alpha s \gamma+c \alpha s \beta c \gamma & x \\
s \alpha c \beta & c \alpha c \gamma+s \alpha s \beta \gamma & -c \alpha s \gamma+s \alpha s \beta c \gamma & y \\
-s \beta & c \beta s \gamma & c \beta c \gamma & z \\
0 & 0 & 0 & 1
\end{array}\right] \\
& { }^{0} X_{H}=\left\{\begin{array}{llllll}
x & y & z & \gamma & \beta & \alpha
\end{array}\right\}^{T}
\end{aligned}
$$

where $c \alpha=\cos \alpha, s \alpha=\sin \alpha$, etc. A vector loop-closure equation is written for each cable (Fig. 11 shows details for the second cable):

$$
{ }^{0} G_{i}+{ }^{0} L_{i}={ }^{0} x_{H}+{ }_{H}^{0} R{ }^{H} h_{i}={ }^{0} H_{i}, \quad i=1,2, \cdots, n
$$

The cable lengths $L_{i}$ are related to the Cartesian pose variables via the lengths (Euclidean norms) of vectors ${ }^{0} L_{i}$ :

$$
\begin{aligned}
& L_{i}=\left\|{ }^{0} L_{i}\right\|=\left\|{ }^{0} H_{i}-{ }^{0} G_{i}\right\| \quad i=1,2, \cdots, n \\
& L_{i}=\left\|{ }^{0} x_{H}+{ }_{H}^{0} R^{H} h_{i}-{ }^{0} G_{i}\right\|
\end{aligned}
$$

Given the cable lengths $\mathrm{Li}$, the forward pose solution calculates the end-effector pose ${ }_{H}^{0} T$. This problem is not straight-forward because it requires the solution of coupled non-linear equations and generally results in multiple solutions. "Analytical" solutions have been presented for similar problems in the past (e.g. [Nanua et.al., 1990]). However, these techniques involve highly complicated symbolic terms and the final result requires finding the roots of a high-order polynomial, which must be performed numerically (for order greater than 4). Therefore, the approach in the current paper is to solve the forward pose problem numerically, applying the Newton-Raphson method to the vector loop closure equations, Eqs. 2. This method yields only one solution, but using the previous solution as the initial guess generally yields convergence to the proper solution.

For CSHIs with actuation redundancy the system of equations is overconstrained. Assuming consistent cable length inputs (i.e. at least one valid forward pose solution exists), these overconstrained equations are beneficial because generally only one solution exists. This eases the problem of multiple solutions. There is an exception to this rule which will be discussed at the end of this section.

Here the forward pose equations are derived and solved for the planar case (Fig. 11). The same method can be extended to the spatial case of Fig. 9. Given $L_{1,2,3,4}$ we must calculate end-effector pose unknowns $x, y, \phi$. Equation 3 is written four times, one for each cable. Each equation is squared to yield four scalar equations in the three unknowns $X=\left\{\begin{array}{lll}x & y & \phi\end{array}\right\}^{T}$ :

$$
\begin{array}{r}
F_{i}(X)=x^{2}+y^{2}+h_{i x}^{2}+h_{i y}^{2}+G_{i x}^{2}+G_{i y}^{2}+2 h_{i x}\left(\left(x-G_{i x}\right) c \phi+\left(y-G_{i y}\right) s \phi\right) \\
+2 h_{i y}\left(\left(y-G_{i y}\right) c \phi-\left(x-G_{i x}\right) s \phi\right)-2\left(x G_{i x}+y G_{i y}\right)-L_{i}^{2}=0 \\
i=1,2,3,4 \quad \ldots(4)
\end{array}
$$

where

$$
\begin{gathered}
{ }^{0} x_{H}=\left\{\begin{array}{ll}
x & y
\end{array}\right\}^{T}, \\
{ }^{H} h_{i}=\left\{\begin{array}{ll}
h_{i x} & h_{i y}
\end{array}\right\}^{T,}, \\
{ }^{0} G_{i}=\left\{\begin{array}{ll}
G_{i x} & G_{i y}
\end{array}\right\}^{T},
\end{gathered}
$$

and the planar orientation matrix is:

$$
{ }_{H}^{0} R=\left[\begin{array}{ccc}
c \phi & -s \phi & 0 \\
s \phi & c \phi & 0 \\
0 & 0 & 1
\end{array}\right]
$$

Equations 4 are solved numerically using NewtonRaphson iteration (e.g. see [Mabie and Reinholtz, 1987, Section 2.3]), modified to handle overconstrained equations. Starting from an initial guess $X_{0}$, the process for step $k+1$ is summarized below:

- $\quad$ Solve $J_{N R} \delta X_{k}=-F(X)$ for $\delta X_{k}$ : 


$$
\delta X_{k}=-J_{N R}^{+} F(X)
$$

- Then $X_{k+1}=X_{k}+\delta X_{k}$

- Iterate until $\left\|\delta X_{k}\right\|<\varepsilon$

where $J_{N R}^{+}=\left(J_{N R}^{T} J_{N R}\right)^{-1} J_{N R}^{T}$ is the overconstrained Jacobian matrix pseudoinverse, $\quad F(X)=\left\{F_{i}(X)\right\}$, $\delta X=\left\{\begin{array}{lll}\delta x & \delta y & \delta \phi\end{array}\right\}^{T}, \varepsilon$ is a user-defined tolerance ( $\varepsilon$ can be different for translational and rotational terms), and the Newton-Raphson Jacobian matrix $J_{N R}$ is:

$$
J_{N R}=\left[\left\{\frac{\partial F_{i}}{\partial x}\right\} \quad\left\{\frac{\partial F_{i}}{\partial y}\right\} \quad\left\{\frac{\partial F_{i}}{\partial \phi}\right\}\right]
$$

where $(i=1,2,3,4)$ :

$$
\begin{aligned}
& \frac{\partial F_{i}}{\partial x}=2\left(x+h_{i x} c \phi-h_{i y} s \phi-G_{i x}\right) \\
& \frac{\partial F_{i}}{\partial y}=2\left(y+h_{i x} s \phi+h_{i y} c \phi-G_{i y}\right) \\
& \frac{\partial F_{i}}{\partial \phi}=2\left(h_{i x}\left[-\left(x-G_{i x}\right) s \phi+\left(y-G_{i y}\right) c \phi\right]+h_{i y}\left[-\left(y-G_{i y}\right) s \phi-\left(x-G_{i x}\right) c \phi\right]\right)
\end{aligned}
$$

This forward pose problem is equivalent to finding the assembly configurations of a four-bar linkage with input/output links $L_{1}, L_{2}$ and two $R R$ constraining dyads of lengths $L_{3}$ and $L_{4}$. By itself the four-bar linkage has infinite assembly configurations because it has 1-dof. $R R$ dyad $G_{3} H_{3}$ constrains the mechanism to a statically-determinate structure of 0-dof. Point $\mathrm{H}_{3}$ defines a four-bar coupler curve which is a tricircular sextic (sixth-degree algebraic curve) that has a maximum of six intersections with the circle of radius $L_{3}$ centered at $G_{3}$ [Hunt, 1990]. $R R$ dyad $G_{4} H_{4}$ further constrains the structure to a staticallyindeterminate structure of freedom -1. Assuming consistent input cable lengths, the circle of radius $L_{4}$ centered at $G_{4}$ must intersect point $\mathrm{H}_{4}$ in one of these possible six solutions. Generally only one of the six possible solutions will assemble with $L_{4}$. The exception to this rule (alluded to above) is that two possible valid solutions exist when the cable connections to ground $G_{i}$ lie on vertices of a square and the hand-grip is a square. The same is true if the ground link and hand-grip are similar rectangles. Therefore, handgrip/ground link symmetry is not desirable as it causes uncertainty in the forward pose solution.

\subsection{INVERSE JACOBIAN MATRIX}

The inverse rate kinematics problem for CSHIs is expressed by:

$$
\dot{L}={ }^{j} M^{j} \dot{X}
$$

where $\dot{L}=\left\{\begin{array}{llll}\dot{L}_{1} & \dot{L}_{2} & \cdots & \dot{L}_{n}\end{array}\right\}^{T}$ is the vector of cable rates, ${ }^{j} M$ is the $n$ by 6 inverse Jacobian matrix expressed with respect to $\{j\} \quad$ coordinates, and ${ }^{j} \dot{X}=\left\{\begin{array}{ll}v & \omega\end{array}\right\}^{T}=\left\{\begin{array}{llllll}\dot{x} & \dot{y} & \dot{z} & \omega_{x} & \omega_{y} & \omega_{z}\end{array}\right\}^{T}$ is the vector of translational and rotational Cartesian rates, also in $\{j\}$. For the planar case, ${ }^{j} M$ is $n$ by 3 and ${ }^{j} \dot{X}=\left\{\begin{array}{lll}\dot{x} & \dot{y} & \omega_{z}\end{array}\right\}^{T}$. The inverse Jacobian matrix ${ }^{j} M$ with $j=0$ may be derived directly as follows (Roberts et.al., 1997). First the $i^{\text {th }}$ cable rate is expressed and related to Cartesian variables using Eq. 3 (where leading superscripts were dropped for brevity):

$$
\begin{aligned}
\dot{L}_{i} & =\frac{d}{d t}\left\|L_{i}\right\|=\frac{1}{\left\|L_{i}\right\|} \frac{d}{d t}\left[\frac{1}{2}\left\|L_{i}\right\|^{2}\right]=\frac{1}{2\left\|L_{i}\right\|} \frac{d}{d t}\left[\left\|x_{H}+{ }_{H}^{0} R h_{i}-G_{i}\right\|^{2}\right] \\
& =\frac{1}{2\left\|L_{i}\right\|} \frac{d}{d t}\left[\left\|x_{H}\right\|^{2}+\left\|{ }_{H}^{0} R h_{i}\right\|^{2}+\left\|G_{i}\right\|^{2}+2 x_{H}^{T}{ }_{H}^{0} R h_{i}-2 x_{H}^{T} G_{i}-2 G_{i}^{T}{ }_{H}^{0} R h_{i}\right] \\
& =\frac{1}{\left\|L_{i}\right\|}\left[\left(x_{H}+{ }_{H}^{0} R h_{i}-G_{i}\right) \bullet v+\left(x_{H}-G_{i}\right)^{T}{ }_{H}^{0} \dot{R} h_{i}\right] \\
& =\frac{1}{\left\|L_{i}\right\|}\left[L_{i} \bullet v+\left(x_{H}-G_{i}\right) \bullet_{H}^{0} \dot{R} h_{i}\right]
\end{aligned}
$$

Working on the rotational term, since ${ }_{H}^{0} \dot{R} h_{i}=\omega \times{ }_{H}^{0} R h_{i}$, we have:

$$
\begin{aligned}
& \left(x_{H}-G_{i}\right) \bullet{ }_{H}^{0} \dot{R} h_{i}=\left(x_{H}-G_{i}\right) \bullet \omega \times{ }_{H}^{0} R h_{i}=\omega \bullet{ }_{H}^{0} R h_{i} \times\left(x_{H}-G_{i}\right) \\
& =\omega \bullet{ }_{H}^{0} R h_{i} \times\left(x_{H}-G_{i}+{ }_{H}^{0} R h_{i}\right)=\omega \bullet{ }_{H}^{0} R h_{i} \times\left(L_{i}\right)=\omega \bullet-\left(L_{i} \times{ }_{H}^{0} R h_{i}\right)
\end{aligned}
$$

where we have used $a \bullet(b \times c)=b \bullet(c \times a)$ and $a \times b=a \times(b+a)$. Now the relationship between one cable rate and the Cartesian rates is:

$$
\dot{L}_{i}=\frac{1}{\left\|L_{i}\right\|}\left[L_{i} \bullet v+\omega \bullet-\left(L_{i} \times{ }_{H}^{0} R h_{i}\right)\right]=\left[\begin{array}{ll}
\hat{L}_{i}^{T} & -\left(\hat{L}_{i} \times{ }_{H}^{0} R h_{i}\right)^{T}
\end{array}\right]\left\{\begin{array}{l}
v \\
\omega
\end{array}\right\}
$$

where $\hat{L}_{i}=\frac{L_{i}}{\left\|L_{i}\right\|}$ is a unit vector in the $L_{i}$ direction. Therefore, the inverse Jacobian matrix is:

$$
{ }^{0} M=\left[\begin{array}{cc}
\hat{L}_{1}^{T} & -\left(\hat{L}_{1} \times{ }_{H}^{0} R h_{1}\right)^{T} \\
\hat{L}_{2}^{T} & -\left(\hat{L}_{2} \times{ }_{H}^{0} R h_{2}\right)^{T} \\
\vdots & \vdots \\
\hat{L}_{n}^{T} & -\left(\hat{L}_{n} \times{ }_{H}^{0} R h_{n}\right)^{T}
\end{array}\right]
$$

This matrix is closely related to the Newton-Raphson Jacobian matrix (given for the planar case in Eq. 6): divide each row of $J_{N R}$ by $2 L_{i}$ to yield ${ }^{0} M$; care must be taken to convert Euler rotational rates to Cartesian rotational rates for the spatial case. To apply Eq. 11 to the planar case, the translational $z$ and rotational $x$ and $y$ terms are dropped because they are zero, resulting in an $n$ by 3 matrix ${ }^{0} M$. 
Kinematic singularities occur at $\mathrm{CSHI}$ configurations where the inverse Jacobian matrix $M$ has less than full rank. Singularities result when $\operatorname{rank}(M)<m$ where $m$ is the dimension of Cartesian space. In the neighborhood of kinematic singularities, the determinant $\left|M^{T} M\right|$ approaches zero. In a serial-chain robotic arm, kinematic singularities physically correspond to configurations where the robot loses one or more degrees-of-freedom. For in-parallelactuated devices such as the Fig. 9 concept, kinematic singularities physically correspond to configurations where the device gains additional, unwanted, uncontrollable degrees-of-freedom.

For planar CSHIs, when the ground link and hand-grip are both squares (or similar rectangles) and $\phi=0$, all configurations are kinematically singular, regardless of translations $x, y$. For cases where the ground link and handgrip are not similar geometric shapes, the only singular configurations are when the hand-grip center is placed at $G_{i} ; i=1,2,3,4$ with $\phi= \pm 90^{\circ}$, which is out of the workspace. The minimum $\left|M^{T} M\right|$ is always greater than zero for non-similar geometric shapes, so there are no singularities in practical operation. Thus, hand-grip/ground link symmetry is not desirable in terms of singularities (this symmetry is also undesirable in the forward pose solution).

\subsection{STATICS MODELING}

This section presents statics modeling for CSHIs. For static equilibrium the sum of external forces and moments exerted on the hand-grip must equal the external wrench exerted on the human. Figure 12 shows the static free-body diagram for the second cable; the remaining seven cables also have tensions $F_{i}$ (not shown for clarity).

The statics equations are:

$$
\begin{array}{r}
\sum_{i=1}^{n} \underline{F_{i}}+m \underline{g}=\underline{F_{R}} \\
\sum_{i=1}^{n} \underline{M_{i}}+{ }_{H}^{0} R^{H} P_{C G} \times m \underline{g}=\underline{M_{R}}
\end{array}
$$

where $F_{i}=-F_{i} \hat{L}_{i}$ is the vector cable tension applied to the $i^{\text {th }}$ cable (in the negative cable length direction $\hat{L}_{i}$ because $\underline{F_{i}}$ must be in tension), $m$ is the hand-grip mass and $\underline{g}$ is the gravity vector, $M_{i}={ }_{H}^{0} R h_{i} \times \underline{F_{i}}$ is the moment due to the $i^{\text {th }}$ cable tension, ${ }^{H} P_{C G}$ is the vector to the hand-grip center of mass from the origin of $\{H\}$, and $\underline{F_{R}}$ and $\underline{M_{R}}$ are the vector force and moment (taken together, wrench) exerted on the human. Moments are summed about the origin of $\{H\}$ and all vectors must be expressed in a common frame, $\{0\}$ in Eq. 12. Substituting the above expressions into Eq. 12 yields:

$$
[A]\{F\}=\left\{W_{R}-G\right\}
$$

where $\{F\}=\left\{\begin{array}{llll}F_{1} & F_{2} & \cdots & F_{n}\end{array}\right\}^{T}$ is the vector of scalar cable forces, $\{G\}=\left\{m \underline{g} \quad{ }_{H}^{0} R^{H} P_{C G} \times m \underline{g}\right\}^{T}$ is the vector of gravity loading, $\left\{W_{R}\right\}=\left\{\begin{array}{ll}F_{R} & M_{R}\end{array}\right\}$ is the external wrench vector exerted on the human by the hand-grip, and the matrix $[A]$ is:

$$
{ }^{0} A=\left[\begin{array}{cccc}
-\hat{L}_{1} & -\hat{L}_{2} & \cdots & -\hat{L}_{n} \\
\hat{L}_{1} \times{ }_{H}^{0} R h_{1} & \hat{L}_{2} \times{ }_{H}^{0} R h_{2} & \cdots & \hat{L}_{n} \times{ }_{H}^{0} R h_{n}
\end{array}\right]
$$

This statics coefficient matrix $A$ is closely related to the inverse Jacobian matrix $M$ of Eq. 11: $A=-M^{T}$.

The statics equations can be used in two ways. Given the cable tensions $\{F\}$ and each $\hat{L}_{i}$ from kinematics analysis, forward statics analysis calculates the external wrench $\left\{W_{R}\right\}$ applied, using Eqs. 12. Inverse statics analysis (calculate the required cable tensions $\{F\}$ given the commanded external wrench $\left\{W_{R}\right\}$ and each $\hat{L}_{i}$ ) is required for tension optimization control so the human can feel $\left\{W_{R}\right\}$ at the hand-grip. This is presented in Section 4.

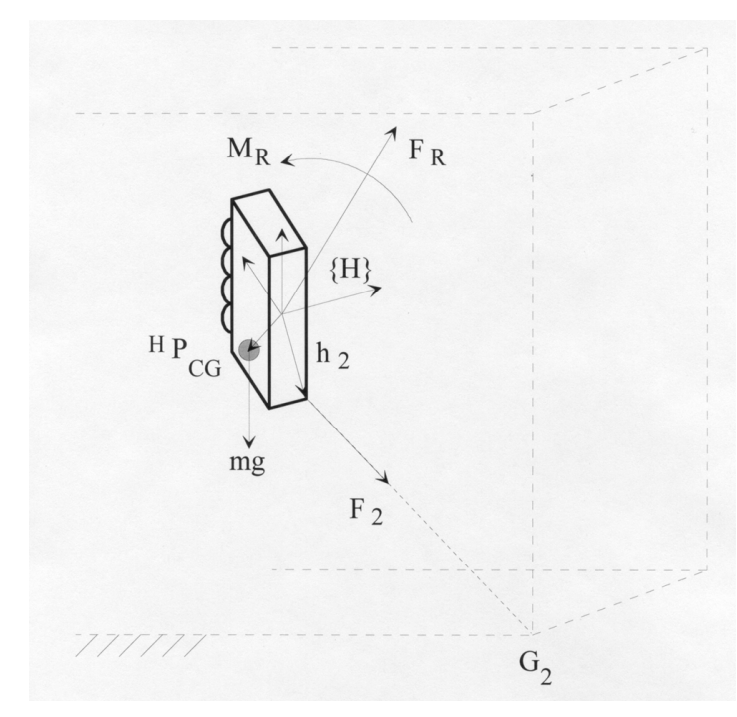

Figure 12. Static Diagram for Second Cable

\subsection{CABLE INTERFERENCE}

The mathematical transformations derived above (forward pose kinematics, inverse Jacobian matrix, statics analysis) assume no cable interference during operation. This is a reasonable assumption when the human inputs do not vary significantly from the equal-cable-length nominal configuration. This would be useful for rate inputs to the virtual world, but position inputs would cause a problem and require extensive re-indexing of the nominal position.

Therefore, for robustness, the kinematics and statics transformations must be updated to include the possibility of 
cable interference. If the cables are sheathed in a lowfriction material, cable interference should present no troubles while extending the useful interface workspace. Modeling for the interference case is a subject for future developments.

\section{Control}

\subsection{GENERAL OPERATION}

Using CSHIs in virtual reality or remote applications, the human motions are sent to the system, while computersimulated or remotely-sensed wrenches must be fed back to the human hand. Each cable requires a length sensor so the forward pose solution can calculate commanded human inputs. Each cable also requires some means for sensing cable tension so closed-loop wrench feedback can be performed. An earlier article by the author [Williams, 1997] presents a general method for Cartesian control of general Force-Reflecting Hand Controllers (FRHCs). These methods are directly applicable to control of CSHIs. However, in order to efficiently apply wrenches in any configuration without permitting any cables to go slack, tension optimization is required for CSHIs.

\subsection{TENSION OPTIMIZATION}

The cable tension optimization problem is stated: minimize $\{F\}$ subject to constraints Eq. 13 and $F_{i} \geq f$ where $f$ is a small positive value. Given the desired external wrench $\left\{W_{R}\right\}$ applied by the hand-grip and each $\hat{L}_{i}$ from kinematics analysis, the required cable forces $\{F\}$ are calculated by inverting Eq. 13. Since cables can only exert tension (and cannot push on the hand-grip), generally actuation redundancy will be required to exert $\left\{W_{R}\right\}$ with no slack cables. That is, we require $n>m$, where $n$ is the number of cables and $m$ is the dimension of the Cartesian space. In this case, Eq. 13 is underconstrained and the general solution for $\{F\}$ is:

$$
F=A^{+}\left\{W_{R}-G\right\}+\left(I_{n}-A^{+} A\right) z
$$

where $I_{n}$ is the identity matrix of order $n, z$ is an arbitrary vector, and $A^{+}=A^{T}\left(A A^{T}\right)^{-1}$ is the underconstrained MoorePenrose pseudoinverse. The first term of Eq. 15 is the particular solution to achieve the desired wrench, and the second term is the homogeneous solution which maps $z$ to the null space of $A$. An equivalent expression for Eq. 15 is:

$$
F=A^{+}\left\{W_{R}-G\right\}+\sum_{j=1}^{n-m} a_{j} N_{j}
$$

where the homogeneous solution is expressed as the sum of (n-m) independent null vectors $N_{j}$ of $A$, multiplied by arbitrary scalars $a_{j}$.
To ensure all cables remain in tension in a given configuration while applying the commanded wrench $\left\{W_{R}\right\}$, $a_{j} j=1, \cdots, n-m$ (alternatively, $z$ ) must be chosen to yield $F$ with strictly positive components. To ascertain the existence of a feasible cable tension vector $F$, Eq. 13 is rewritten as:

$$
\bar{A} \bar{F}=0
$$

where $\bar{A}=\left[\begin{array}{ll}A & G-W_{R}\end{array}\right]$ and $\bar{F}=\left\{\begin{array}{ll}F & 1\end{array}\right\}^{T} \cdot \bar{F}$ must be in the left null space of $\bar{A}$ in order to satisfy Eq. 17 . An algorithm for ascertaining the existence of a feasible solution to Eq. 17 (or Eq. 13) is given in Roberts et.al. [1997]. That work was motivated by the Charlotte application at NASA KSC. Roberts' method is summarized below:

Static equilibrium is possible at a given configuration if and only if a left null vector of $\bar{A}$ exists with all components non-negative and last component nonzero.

The $i^{\text {th }}$ cable tension is given by the ratio of the $i^{\text {th }}$ and last component of a non-negative left null vector of $\bar{A}$ satisfying the above statement. Any zero components correspond to slack cables. If the last component of every null vector is zero, the last column of $\bar{A}$ is not in the column space of $A$ so the hand-grip cannot achieve static equilibrium in that configuration even if the cables could push. Not all CSHI configurations can exert any desired external wrench. The author and his students are currently researching the wrench-exerting limitations for general CSHIs.

\section{VR Applications}

The cable-suspended haptic interface $(\mathrm{CSHI})$ may be used as a spatial 6-dof input/output device for general virtual reality (VR) applications. The CSHI is used for input when the user moves the hand-grip to explore virtual environments. It is used for output when reflecting sixdegree-of-freedom wrench feedback to the operator's hand from the virtual environment. The potential areas for haptic implementation in VR in the research, education, and commercial sectors is very broad, including computergenerated scientific visualization, flight simulations and actual flight missions, virtual training, virtual collaborative environments, rapid prototyping and virtual manufacturing, telerobotics, biomedical visualization, surgical training, remote surgery, education, and entertainment. Haptics adds a major dimension (force/moment and tactile information) missing in many current VR implementations focusing on graphics and audio feedback. Inclusion of the haptic sensory feedback mode can greatly improve telepresence quality. 
One exciting area for future development with CSHIs is wrench/tactile fusion. That is, different tactile feedback elements can be included at the hand-grip to provide simultaneous force/moment and tactile feedback. Some early work has been accomplished in this area (e.g. [Kontarinis and Howe, 1995; Chen and Marcus, 1994], but it tends to focus on one-degree-of-freedom force feedback and a specific tactile element. The bulk of the haptics research and development work to date focuses on either wrench or tactile feedback. Wrench/tactile fusion is a rich area for future VR haptic feedback research involving both mechanical/computer developers and human factors researchers. The CSHI will be a tool in this pursuit.

\section{Conclusion}

A cable-suspended haptic interface $(C S H I)$ concept was presented. Though the focus was wrench reflection, future plans include wrench/tactile feedback fusion using the same device. The forward pose solution, Jacobian matrix, statics equations, and tension optimization algorithm were discussed. Cartesian control methods in an earlier article by the author may be implemented for control of general CSHIs in virtual reality and remote operations. A planar CSHI prototype with four cables has been completed and a spatial CSHI prototype with eight cables is under development.

\section{Acknowledgements}

This research was supported in part by an Air Force grant and the AFOSR Summer Faculty Research Program. The author acknowledges researchers in the Human Sensory Feedback Lab at Wright-Patterson AFB for support and facilities.

\section{REFERENCES}

J.S. Albus, R. Bostelman, and N.G. Dagalakis, 1993, "The NIST ROBOCRANE", Journal of Robotic Systems, 10(5): 709-724.

P.D. Campbell, P.L. Swaim, and C.J. Thompson, 1995, "Charlotte Robot Technology for Space and Terrestrial Applications", 25 $5^{\text {th }}$ International Conference on Environmental Systems, San Diego, SAE Paper 951520.

E.Y. Chen and B.A. Marcus, 1994, "EXOS Slip Display Research and Development", ASME International Mech Engr Congress, Chicago, IL, DSC 55(1): 265-270.

J.J. Craig, 1989, Introduction to Robotics: Mechanics and Control, Addison Wesley Publishing Co., Reading, MA.

K.H. Hunt, 1990, Kinematic Geometry of Mechanisms, Clarendon Press, Oxford.

M. Ishii and M. Sato, 1994, "A 3D Spatial Interface Device Using Tensed Strings", Presence-Teleoperators and Virtual Environments, MIT Press, Cambridge, MA, 3(1): 81-86.
D. Kontarinis and R. Howe, 1995, "Tactile Display of Vibratory Information in Teleoperation and Virtual Environments", Presence-Teleoperators and Virtual Environments, MIT Press, Cambridge, MA, 4(4): $387-$ 402.

R. Lindemann and D. Tesar, 1989, "Construction and Demonstration of a 9-String 6-DOF Force Reflecting Joystick for Telerobotics", NASA International Conference on Space Telerobotics, (4): 55-63.

H.H. Mabie and C.F. Reinholtz, 1987, Mechanisms and Dynamics of Machinery, Wiley, NY.

T.H. Massie and K.J. Salisbury, 1994, "PHANToM Haptic Interface: A Device for Probing Virtual Objects", ASME International Mech Engr Congress, Chicago, IL, DSC 55(1): 295-299.

P. Nanua, K.J. Waldron, and V. Murthy, 1990, "Direct Kinematic Solution of a Stewart Platform", IEEE Transactions on Robotic and Automation, 6(4): 438443.

R.G. Roberts, T. Graham, and J.M. Trumpower, 1997, "On the Inverse Kinematics and Statics of Cable-Suspended Robots", IEEE International Conf. on Systems, Man, and Cybernetics, Orlando, FL.

D. Stewart, 1966, "A Platform with Six Degrees of Freedom", Proceedings of the Institute of Mechanical Engineers (London), 180(15): 371-386.

R.L. Williams II, 1997, "Control of Kinesthetic Haptic Interfaces in VR Applications", International Journal of Virtual Reality, 3(1): 18-26.

\section{BIOGRAPHY}

Robert L. Williams II (photo on CDROM) is currently assistant professor of Mechanical Engineering at Ohio University in Athens. His present research interests include control of telerobotic systems, virtual reality, and haptic interfaces. Recently Dr. Williams has been collaborating with researchers in the Human Sensory Feedback Laboratory at Wright-Patterson AFB on various haptics and telerobotics projects. Previously Dr. Williams worked at NASA Langley Research Center in the control of telerobotic systems for remote space applications.

\section{Contact information:}

Robert L. Williams II

Dept. of Mechanical Engineering

257 Stocker Center

Ohio University

Athens, OH 45701-2979

Phone: 740-593-1096

FAX: 740-593-0476

Email: bobw@bobcat.ent.ohiou.edu 